\title{
Desde la acción a la enacción. Más allá del movimiento y de la Educación Física*
}

\author{
From the action to enaction. Beyond of the movement and the Physical Education \\ Da ação à contra ação. Além do movimento e da educação Física
}

\author{
Sergio Toro Arévalo, Pamela Valenzuela Mautz ${ }^{\mathrm{a}}$ \\ ${ }^{a}$ Facultad de Filosofía y Humanidades, Universidad Austral de Chile, Instituto de Filosofía y Estudios \\ Educacionales. Correo electrónico: seatoro@gmail.com
}

\begin{abstract}
RESUMEN
El presente trabajo pretende entregar una visión general acerca de los principios que sustentan la acción como fenómeno ecológico, favoreciendo una comprensión diferente de lo humano. Se ha optado por un enfoque sistémico y complejo, en función de distinguir que la unidad básica de la motricidad humana está precisamente en la acción, diferenciándose del concepto tradicional de movimiento imperante en la educación física actual. En tal esfuerzo, se consideran aproximaciones y propuestas provenientes de campos diferentes como la psicología, filosofía y neurología, que discuten diversos insumos categoriales y conceptuales, desencadenado una revisión o, por lo menos, una consideración y actuación disciplinar diferente a lo que comúnmente se realiza en el campo de desempeño profesional, tanto en el ámbito epistemológico como metodológico. Como consecuencia, nos invita a superar categorías y principios de comprensión paradigmática y despliegue didáctico.
\end{abstract}

Palabras clave: acción, motricidad humana, conocimiento, aprendizaje.

\begin{abstract}
The following paper pretends to give a general vision about the principles that support the phenomenological ecology as an action that enhances a different human comprehension. We opted for a systemic and complex focus in terms of distinguishing the basic unit of human movement, more precisely in the action, differing from the traditional concept of movement prevailing in the current physical education curricula. In this effort, proposals are considered from different fields such as psychology, philosophy and neuroscience, in order to discuss various categorical and conceptual inputs, creating a review or at least a disciplinary consideration and action different from others, which are currently done in the professional field, as epistemological and methodological ones, and it even invites us to overcome categories in paradigmatic and didactic deployment.
\end{abstract}

Key words: action, human motricity, knowledge, learning.

\section{RESUMO}

Objetiva-se dar uma visão panorâmica acerca dos princípios que sustentam a ação como fenômeno ecológico que favorece a diferença na compreensão do ser humano. O enfoque é sistêmico e complexo a fim de distinguir que a unidade básica da motricidade humana está precisamente na ação, em detrimento do conceito tradicional de movimento predominante na Educação Física atual. Nesse sentido, consideram-se aproximações e propostas provenientes de

* $\quad$ Este artículo fue solicitado por la Revista Estudios Pedagógicos en diciembre del 2011 en el contexto del proyecto de Investigación FONDECYT (Fondo de Investigación Científica y Tecnológica) № 11110016 , titulado "Educación Física y su función de transformación de las desigualdades sociales: profesorado del área y documentación ministerial". El artículo fue aceptado en junio de 2012. Asimismo, el texto desarrollado en contexto del proyecto Fondecyt 1120761, titulado Conocimiento y comunidad escolar: procesos de interpretación de las dinámicas relacionales escolares en la educación básica como configuración cognitiva situada. 
diferentes campos, como psicologia, filosofia e neurologia, que discutem diversas correntes categoriais e conceituais, gerando uma revisão, ou no mínimo, uma consideração e atuação na área diferente do que se concretiza no campo do desempenho profissional, tanto no âmbito epistemológico como metodológico e que, inclusive, convida-nos a superar categorias e princípios de compreensão paradigmática e desdobramento didático.

Palavras chave: ação, motricidade humana, conhecimento, aprendizagem.

\section{PRESENTACIÓN}

Las palabras son dueñas del espacio
pero también esclavas de los necios
tienen la impavidez de los que mandan
pero también en ellas se sostiene
el corazón de las transformaciones.

Mario Benedetti (2009)

En la actualidad parece no haber duda que lo que se conoce como Educación Física es un "campo de estudio" validado y establecido que no requiere de discusión. Mas aún, si se considera el crecimiento explosivo en términos de oferta para estudiar en Chile (mas de 80 programas se ofrecen actualmente), según los criterios de mercado, tan relevantes en los procesos de argumentación e interpretación de la situación y los fenómenos que se presentan. Este aspectos resulta relevante si consideramos que los procesos de acreditación se han orientado hacia la confirmación, intencionada o no, consiente o no, de determinadas aproximaciones epistemológicas y paradigmáticas que se presentan en nuestra sociedad. El ejemplo más claro de dicho proceso, por lo menos en el caso chileno, lo constituye lo denominado como SIMCE (Sistema de Evaluación de la Calidad de la Educación) de Educación Física, que pretende evaluar capacidades condicionantes como la fuerza, la resistencia aeróbica y la composición corporal, más que aprendizajes generados desde la acción y relación didáctica configuradas en las clases.

En este sentido, la educación física, desde nuestro parecer, se comporta como una ideología o dinámica doctrinaria, al decir de Zîzêk (2011), dado que se funda sustantivamente en una lógica de actividad, restando un componente reflexivo que se sustente más allá de la disciplina y que, al mismo tiempo, le permita consistencia y coherencia interna de base, en su desarrollo y diálogo con el mundo que le rodea. Además, que dé cuenta y anticipe ciertas dinámicas de desenvolvimiento personal y propuestas hacia la cultura. Pero, ¿cómo dar cuenta de una disciplina que tiene una presencia tan variada y extensa, y al mismo tiempo lejos de una cierta presencia y consolidación social desde el punto de vista académico y cultural? Es decir, ¿cómo identificar un componente común que exprese la unidad e identidad profesional del mismo quehacer, más allá de las aproximaciones terminológicas, lingüísticas e incluso paradigmáticas?

En tal sentido, cabe preguntarse: ¿Cuál es aquel objeto sobre el cual se sitúa el desempeño profesional de un experto que se desenvuelve en el contexto educativo, deportivo, recreativo, fitness, desarrollo comunitario, promoción de la salud, de hábitos saludables, de diferentes edades y condiciones? De igual manera, ¿cómo es posible tanta flexibilidad de campo laboral sin contradicciones éticas ni legales? ¿sobre cuáles fundamentos se justifican o se comprende la pertinencia tanto que el profesional hace de su propia labor, como la sociedad que lo fortalece y valida? ¿Cuál es la base común que permite tanto la justificación como la diversidad de esta situación? 
En consecuencia, se hace cada vez más evidente la necesidad de. por lo menos. iniciar la discusión sobre aquello que se comparte y sobre qué se define la orientación y distinción del desempeño que realizan dichos profesionales. Sobre todo, que tal esfuerzo permitiría evaluar con mayor claridad la pertinencia del mismo proceder o desempeño profesional.

Comenzaremos señalando que desde perspectivas clásicas se sigue sosteniendo que la base del quehacer de un profesional de la Educación Física es la educación a través y por el movimiento. Desde nuestro enfoque, dicho planteamiento nos resulta altamente insuficiente, como lo hemos discutido en textos anteriores (Sergio y Toro, 2004; Toro, 2010; Toro, 2009). En el presente trabajo, más bien, se pretende entrar en la discusión y desarrollo de la opción que si se comparte y se intenta llevar a cabo. Incluso, procurando desplegar un lenguaje que permita claridad y al mismo tiempo coherencia epistémica y programática. De manera que el entendimiento de la acción se convierte en el eje de desarrollo y objetivo del presente esfuerzo.

El primer acercamiento se configura desde la filosofía; posteriormente, iremos abarcando aportes desde la psicología y las neurociencias para confluir en la propuesta presentada por Francisco Varela $(1997 ; 2000)$ denominada Enacción.

\section{LA ACCIÓN COMO PLATAFORMA DE SER HUMANO}

Porque en toda acción, lo que intenta principalmente el agente, ya actúe por necesidad natural o por libre voluntad, es explicar su propia imagen. De ahí que todo agente, en tanto que hace, se deleita en hacer; puesto que todo lo que apetece su ser, y puesto que en la acción el ser del agente está de algún modo ampliando, la delicia (Cit. en Arendt, 1993:199)

Uno de los aspectos más radicales en la propuesta de trabajo de Hanna Arendt está en comprender, en la acción, la distinción central de lo humano, tanto así como en la palabra. Es en la acción que cada sujeto despliega su particularidad y distinción, no obstante, se da siempre en la paradoja de la pertenencia a una determinada especie, en primer lugar, y una condición, lo vivo, en segundo. No podríamos conceptualizar lo que cada uno de nosotros hace si no consideramos que en el proceso de la acción es donde cada cual expresa y al mismo tiempo impresa su propia potencialidad.

actuar, en su sentido más general, significa tomar una iniciativa, comenzar (como indica la palabra griega archein, "comenzar", "conducir" y finalmente "gobernar"), poner algo en movimiento (que es el significado original del agere latino)" . debido a que son initium los recién llegados y principiantes, por virtud del nacimiento, los hombres toman la iniciativa, se aprestan a la acción" (Ibíd., 201).

En la acción el ser humano emprende su propia existencia; allí es donde se constituye y consolida el ser, desplegando en cada acto su capacidad y también su limitación. Pero es cada vez un inicio, una apertura, aunque la acción haya sido realizada muchas veces antes. Su cualidad se centra en la libertad de acceder al acto.

Mediante la acción y el discurso, los hombres muestran quienes son, revelan activamente su única y personal identidad y hacen su aparición en el mundo humano...esta cualidad reveladora del discurso y la acción pasa a primer plano cuando las personas están con otras, ni a favor ni en contra, es decir, en pura contigüidad humana (Ibíd., 203-204). 
Es aquí, en la acción como lugar y discurso, donde el ser humano ocurre. Desde las perspectivas más racionales, como el Dasein heideggeriano, el hombre en el mundo de Merleau-Ponty, el complexus de Morin (1992) y, porqué no decirlo, en las cosmovisiones de los pueblos indígenas, en la acción desplegada es que tiene lugar lo humano en su sentido de fondo y profundidad. Pero queda remarcado que tal acción es precisamente en vínculo continuo con la especie, la comunidad y el entorno, no en la negación de estos factores.

En este proceso se genera toda la posibilidad humana, no como una suma de partes, sino en una especie de relación de actores y actuantes que confluyen en cada demanda o tarea que se genera en el entorno. Ya nos dice Fuchs (2010) que desde el comienzo de la gestación de un ser humano y utilizando la comunicación afectivo-encarnada, se genera lo que ha denominado como memoria interactiva producida en todos los niveles del o la recién nacido/a. Es decir, no existiría un desarrollo particular de cada dimensión de un ser humano, por el contrario, se produce en igual medida lo senso-motor, emocional y temporal. Lo fundamental de esta situación es que en la acción en curso lo que genera la correspondiente evolución de los mismos componentes que la posibilitan, en palabras de Kandel (2003), es ser humano está hecho para la acción y debemos agregar que también se hace desde la acción.

No obstante, dicho proceso no ocurre en un vacío existencial ni menos temporoespacial, es decir, está situado, implicado e inmerso en un contexto geográfico, simbólico, sentimental, histórico y cultural. Es una relación desde una agente en un entorno que al mismo tiempo es generante y condicionado. Es en palabras de Varela, Thompson y Rosch (2005) un acoplamiento estructural, o un vínculo de resonancia recíproca o configuración mutua. En otras palabras, al actuar nos configuramos tanto como seres y como entorno. Tal aproximación en un cambio radical en lo que se ha entendido normalmente por inteligencia y cognición, como también lo que se ha entendido por motor o movimiento.

Si lo llevamos al contexto del aprendizaje, por ejemplo, deberíamos concordar con Freeman (2007), quien sostiene que lo que se aprende no son estímulos sino contextos, y de igual forma la acción aprendida no sería un despliegue biomecánico ciego, por el contrario, está inundada de intencionalidad y simbolismo. En ese sentido, se hace comprensible que el comportamiento esté anclado en la experiencia de valorarse a sí mismo en determinados contextos de ejercicio. De manara que es muy normal que nuestro comportamiento se modifique bastante dependiendo del lugar y momento en que nos encontramos, incluso a pesar de tener o haber desarrollado una práctica o entrenamiento sostenido en situaciones parecidas o similares. La experiencia siempre será contingente y situada (James, 2009).

Por otra parte, la acción, como concepto, se aleja de la conducta en virtud de los orígenes terminológicos, epistemológicos y, por lo tanto, paradigmáticos de la conducta, lo cuales refieren principalmente al comportamiento observable y externo del sujeto y deja de lado los componentes internos presentes, ${ }^{1}$ gravitantes en el proceso y resultado

\footnotetext{
El referente más sostenido de esta postura es el conductismo desarrollado primeramente por J. Watson, A. Pavlov y posteriormente por Skinner. Esta corriente, dada su popularidad y "eficiencia" en la modificabilidad de la conducta, tuvo un gran difusión en los sistemas educativos y clínicos, a través de los procesos de condicionamiento. Cabe señalar que lo fundamental de dicha escuela es sostener que lo fundamental dentro del comportamiento humano es precisamente la conducta observable, no aquello que se encuentra dentro de la "caja negra" que podemos definir como conciencia o intencionalidad.
} 
del comportamiento. Bernstein (Cit. en Batalla Flores, 2005), uno de los autores más referenciado dentro del estudio disciplinar, señalaba en respuesta a las visiones reactivas y conductistas de la motricidad humana, que precisamente lo que definimos como "movimiento humano "está impreso de objetivos, que se desencadenan hacia la solución de problemáticas que se presentan en la cotidianidad de la vida humana. Por lo tanto, está muy lejos de un mero dislocamiento o reacción subsidiaria de estímulos externos, de manera que su comprensión y estudio demanda la atención sobre procesos que se expresan tanto internamente dentro de los sujetos como de aspectos que se contextualizan en el entorno que se desenvuelven".

Dichos planteamientos, realizados en el siglo pasado, hoy parecen tener su relato actual en lo que nos presenta Rizzolatti (2006), basado en sus estudios sobre la relaciones neurológicas entre el sentir y el operar, al plantear:

Todo lo cual no hace sino confirmar la interdependencia de la constitución de los objetos y del espacio que resulta de la referencia común al horizonte primordial de la acción... una vez mas, aparecen muy claros los limites de cualquier interpretación rígidamente dicotómica sobre el funcionamiento de nuestro cerebro, como aquellas que se basan en la contraposición entre la vía de qué y la vía del dónde, o también entre la vía del qué la vía del cómo (Rizzolatti, 2006:83).

Por lo tanto, al adentrarnos en lo que el "Humanes" (Zubiri, 1986) realiza, no se puede reducir a un movimiento o una conducta, toda vez que su hacer contempla otros elementos que sobrepasan y superan lo que ambos conceptos comprenden. En tal sentido, compartimos la visión de Manuel Sergio (1999) quien, desde una visión epistémica compleja, nos plantea la motricidad como intencionalidad operante, vale decir, acción. Del mismo modo, se comparte la propuesta antropológica del autor, al plantear la irrenunciable tarea de comprender al ser humano como una unidad multidimensional indivisible.

Tal aproximación nos plantea la exigencia de considerar lo que Schütz (1995) define como el Lebenswelt o mundo de la vida, en lugar de priorizar situaciones artificiales, experimentales o extremadamente especiales o inusuales. Entrar en lo que hacemos cada día comprende, precisamente, relevar las actividades de carácter inmediato y cotidiano que le dan un sentido y dirección a la forma de vida que se lleva a cabo. De manera que levantarse, preparar el desayuno, dirigirse hacia algún lugar en forma permanente o esporádica, tomar locomoción, pagar, etc., etc., son acciones que van definiendo nuestros proyectos o formas de vida, desde lo más incipiente y banal hasta los más profundo y trascendental. Pero estas acciones se producen dentro del marco de operaciones que al ser humano en tanto sistema biológico-cultural en primer lugar le es posible realizar. Dentro de estas posibilidades está la percepción como una características básica que permite entrar en relación con el entorno y su especie. Constituyéndose de la misma forma en la percepción en un movimiento intencionado o guiado perceptualmente (nuevamente, acción) hacia el acoplamiento con el entorno (Varela, 2000).

Desde esta particularidad es que el ser humano desenvuelve su vida en construcción y contacto con los intencionalidades y propósitos que orienten sus acciones en el espaciotiempo pleno de otros sentidos y significaciones, ampliando la relación y posibilitando el transito desde el espacio-tiempo local a lo histórico-cultural, y desde aquí construye el mundo. Situación que sólo se produce en sociedad, en una relación fluida de dependencia - autonomía de los otros sujetos y de otras especies. 
De manera que la acción, entendida como el despliegue de la intencionalidad, propósito y significancia adquiere una dimensionalidad distinta, y tiene lugar en la trama de identidades sin un centro específico (Varela, 2000). Estas identidades alcanzan su sentido en la relación dinámica y recíproca que ellas mismas generan y de esta misma forma se generan a sí mismas en forma organizada y autorregulada (principio de autopoiesis de Maturana y Varela, 1996). A partir de esta relación, de base biológica, es que el ser humano entre en relación con él mismo, con los demás y el entorno conformando un mundo no tan sólo biológico, sino también cultural, en una relación permanente e indivisible, desde y en su experiencia constante.

De esta forma la acción responde no sólo a la consecución del propósito sino también a las características del actor y del entorno en el cual actúa. De manera que se hace necesario adentrarse en los aspectos sicológicos y sociales que el actuar nos plantea, desde el punto de vista descriptivo, conceptual y operativo.

Pero, en definitiva, cuáles son las estructuras conceptuales que permiten aproximarnos a una teoría de la acción desde el punto de vista de la psicología desde una mirada respetuosa de la propuesta epistemológica asumida, vale decir fenomenológica (mundo de la vida). Para la consecución de esta tarea, nos hemos propuesto plantear los aspectos fundamentales de la acción desde diversos autores (Gabler, 1996; Vayer y Roncin, 2000; Merleau-Ponty, 2004; Freeman, 2007), como también desde distintos aportes de otras disciplinas que observan y desarrollan estos elementos desde miradas complementarias, a saber, la neurociencia (Noe, 2010; Fuchs, 2010; Rizzolatti, 2006; Changeux, 2003; Damasio 2010), la biología (De Waals, 2010, Maturana, 1999) y la sociología de la acción (Luckmann, 1997; Schutz, 1995).

\section{FUNDAMENTOS DE LA PSICOLOGÍA DE LA ACCIÓN}

La psicología de la acción plantea una forma de comprender, de investigar y proceder en lo que refiere al comportamiento humano (Gabler, 1996), es decir, implica entender que el ser humano, a partir de la acción, se despliega en el entorno de acuerdo, como ya se ha dicho, a determinadas intenciones y propósitos que el actor/a genera o hace emerger y vivencia de acuerdo a su contexto socio-cultural-natural. Esto comprende la actuación y comportamiento del que investiga y realiza una determinada praxis basado en la teoría de la acción.

Si se considera la teoría de la acción como una perspectiva general para el desarrollo de un modelo psicológico, una metodología de investigación y una praxis, es necesario entender cuáles son sus fundamentos y sus más esenciales principios.

Seguidamente intentaremos exponer en forma clara los principios y fundamentos de esta teoría. Se entiende la perspectiva de la teoría de la acción a través de cuatro fundamentos o postulados (Gabler, 1996; Vayer y Roncin, 2000), a saber:

3.1. Postulado sistémico: el actuar es entendido, desde una perspectiva general, como un proceso integral y complejo donde intervienen una gran cantidad de elementos interrelacionados entre sí, es decir como un proceso sistémico cuyo propósito central se orienta hacia la configuración relacional entre sujeto y entorno. Asumiendo de suyo que cada uno de estos constituyen sistemas en sí mismo, dispuestos y orientados desde y hacia la relación potenciadora y al flujo dinámico de energía y de contingencia. 
3.2. Postulado de la intencionalidad: el actuar es entendido como una forma particular del comportamiento en dirección o flujo, como una inversión y conversión de energía que se orienta, nortea y dirige en múltiples sentidos y que simultáneamente se afecta a si misma, es decir como intencional. La intención es el principio generador a través del cual se produce el comportamiento, pues motiva a la consecución de la misma intención o del propósito que una persona configure. La intencionalidad se produce o posee dos vertientes, por un lado, está la necesidad de satisfacer las carencias que cada ser humano tiene dentro de su característica multidimensional. Complementariamente, la intencionalidad se genera desde la emoción, en el sentido de un fenómeno múltiple (fisiológico, químico, psicológico) que produce un cambio de disposición de la persona. La intencionalidad ocurre dentro de un determinado marco cultural, se desprende y al mismo tiempo ocurre en la cultura en la cual el sujeto se desenvuelve. La vivencia cotidiana de las personas es la conciencia de la intencionalidad de la situación o objeto vivenciado, entendiendo objeto en el sentido genérico (Dantas, 1999). Tal aspecto se impresa y expresa en marcadores somáticos (Damasio, 1999, 2010) irrenunciables que definen nuestro sentir en la contingencia vivida y por ende su carga significante.

3.3. Postulado de la regulación: el actuar, como un comportamiento intencional no sólo se puede explicar a partir de mecanismos de regulación biológica sino que es esencialmente regulado en la psiquis, entendida como el relato histórico-encarnado de lo vivido en el que confluyen procesos pre-reflexivos y reflexivos propiamente tal, siempre presente y siempre actualizándose. Por lo cual desde allí se organizan primeramente los sentidos de las distinciones que una acción determinada puede producir, produce o produjo, de acuerdo a la consecución o no del propósito de la acción. Del mismo modo, actúan mecanismos sociales que dan curso y/o definen una determinada acción de acuerdo a los factores culturales particulares de cada grupo o sociedad.

3.4. Postulado del desarrollo: la psiquis se desarrolla en y desde el actuar, y el actuar es en su condición general un proceso sistémico. En su intencionalidad y en sus bases de regulación es también un fenómeno filo-ontogenético, en el sentido que depende de las capacidades propias del sujeto perceptor dentro de un contexto de especie y de individuación, pero al mismo tiempo es de acuerdo a su marco de condiciones de vidas sociales un fenómeno sociocultural e histórico. En este sentido, el desarrollo presenta tres niveles, a saber: lo filogenético, lo ontogénico y el aquí y ahora (Pfeifer y Bongard, 2007). De modo que los procesos que convergen en una acción se originan de la concurrencia de estos tres afluentes, de esta situación se puede desprender que el desarrollo es permanente y, por consecuencia, la evolución también, aunque en el día a día no se pueda apreciar con mucha claridad. Por otra parte, también se puede desprender que el desarrollo más que una sucesión de etapas, es cercano a los patrones culturales que en una determinada sociedad se generen. Es decir la acción humana, en virtud de su desarrollo se situará socio-culturalmente en función de las formas y modos de relación que emplea.

\section{EL ACTUAR COMO UN PROCESO SISTÉMICO}

La expresión del comportamiento como un proceso sistémico significa que la acción tiene que ver con un sistema complejo y dinámico (Figura 1), en el cual sus principios de organización están constantemente siendo convocados y requeridos. Desde este 
planteamiento, la acción se observa y considera como el sustrato del comportamiento humano, caracterizado como un proceso en el cual intervienen una gran variedad de elementos, consideraciones y dimensiones que deben precisamente ser comprendidos e interpretados en la densidad y condiciones de las relaciones que entre ellos se producen, pues en ellas se define y tiene lugar la acción. Esto no significa que no se pueda distinguir, en detalle, elementos que conforman el sistema, como por ejemplo: percepción, pensamiento, motivación, emoción, entre otros. Desde el mismo modo, también puede estudiarse y analizarse las dependencias y correspondencias entre dos o más elementos en forma particular, pero se corre el riesgo tremendo de caracterizar los elementos y caer en la ilusión que existen en sí mismos o constituyen identidades objetivas y por lo tanto no entender el sistema como tal. Esta situación está muy difundida en el quehacer educativo en particular y en la sociedad en general. Específicamente en el contexto de la educación escolar se entiende el aprendizaje como una acción parcelada por disciplinas, y dentro de ellas se separa o escinde el mismo proceso de aprendizaje reduciéndolo al escuchar o al repetir lo que los docentes expresan.

Es en la relación y coordinación de los diversos elementos la que permite el actuar y, por ende, desde la psicología o desde cualquier intervención o potenciamiento del actuar, se debiera estudiar y analizar el comportamiento. Esta ultima expresión comprende amplias y prácticas consecuencias; a manera de ejemplo, una enfermedad sería entendida no como la falla de función de un órgano en particular o de la persona en total, sino primeramente como una falla en la función de una persona enferma en y a través de su actuar en un determinado marco histórico cultural, en el que se despliega la propia vida del enfermo. En el mismo sentido sería asociable al entrenamiento no sólo sobre una dimensión particular (corporal o psicológica), sino sobre un determinado contexto y de la globalidad del actuar de la persona en ese contexto.

Además, esta perspectiva valora el impacto que tiene el entorno, sea material o social sobre la persona en sus distintas dimensiones (psicológica, corporal, espiritual), esto quiere decir que el proceso sistémico del actuar también comprende los elementos que se encuentran "fuera" de la persona y que a su vez se relacionan con los procesos internos (dimensión objetiva y subjetiva respectivamente), y que en conjunto producen el actuar o la acción (Figura 1).

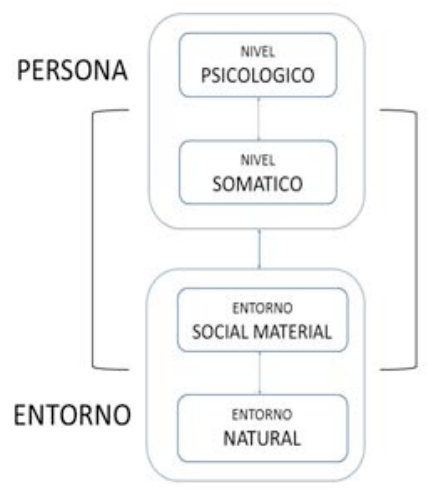

Figura 1. Componentes de la acción. (Gabler, 1996) 
Gabler (1996) caracteriza esta postura como una visión ecológica de la teoría de la acción, pues consiste en una confluencia y relación de una persona con su correspondiente entorno social y/o material. En tal sentido la acción es una unidad dinámica entre la persona y el entorno. Por ejemplo, al realizar una acción se conjugan dos dimensiones en la realización de la misma: las dimensiones subjetiva y objetiva. En cada realización de una tarea concurren elementos propios de la subjetividad de la persona como son su propia percepción, interpretación y significancia que le da a la realización de la tarea. Cabe señalar que este proceso es individual y depende de la historia del individuo. Al mismo tiempo concurre la dimensión objetiva que es independiente de la percepción e interpretación del individuo y se relaciona con los acuerdos y consensos sociales sobre el tiempo-espacio y la tarea que tiene lugar en la acción. Por ejemplo, aprobar una determinada asignatura en el colegio o cumplir con una determinada calificación o rendimiento, es objetivo para todos los alumnos, como también que éste último (persona) tiene o debe aprobar; en este caso el entorno está representado por el ambiente escolar. La dimensión subjetiva comprende los mismos elementos, pero situados desde interpretación de la persona en cuanto a la densidad significativa que cada aspecto en tiene desde la vivencia particular.

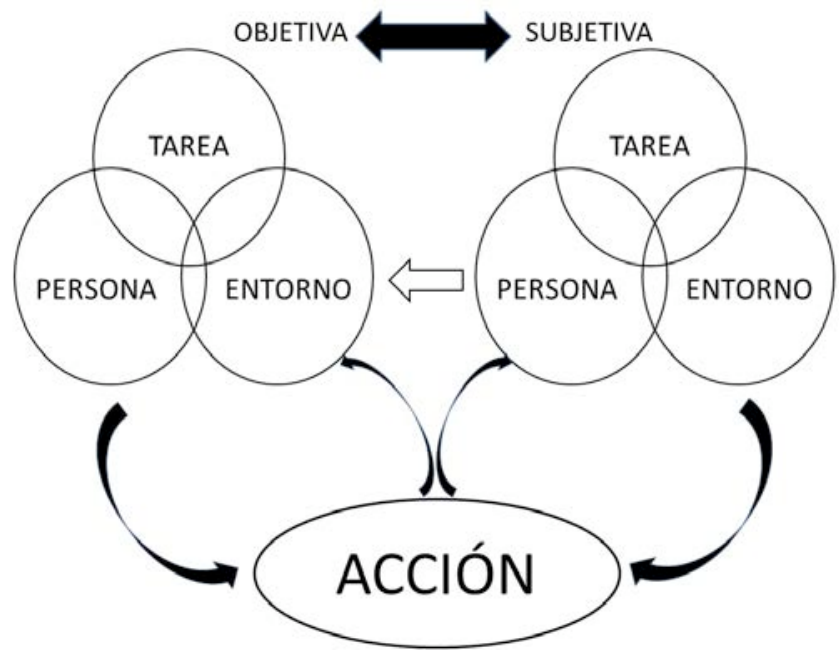

Figura 2. Dimensiones de la acción (Gabler, 1996)

Es importante señalar que la unidad del proceso de acción como sistema también se produce en la relación de la corporalidad y la psiquis, es decir, la acción es al mismo tiempo un suceso somático y psíquico. No es posible en la acción humana separar estos dos aspectos, como tampoco determinar donde comienza uno y termina el otro, por el contrario, los dos aspectos representan dos facetas de una misma realidad.

No obstante lo fundamental, desde dicho planteamiento, se base en que cada acción de suyo, es un proceso intencional, aspecto que revisaremos a continuación. 


\section{EL ACTUAR COMO COMPORTAMIENTO INTENCIONAL}

En un sentido amplio, el comportamiento es entendido en relación a las características de expresión de un sistema dinámico. Orientado hacia los seres vivos, se define como cada forma de expresión de vida, es decir, la característica de expresión permite entrar en relación con el entorno y modificarlo. Pero dicha modificación es precisamente producto de un diálogo entro lo impresivo y expresivo de la vida, es decir desde el punto de vista biológico, en primer lugar, y que posteriormente se amplía y profundiza desde lo socio-cultural. En otras palabras, lo primero que se intenciona se genera desde los procesos de regulación metabólica, desde lo fundamentalmente biológico. Por ejemplo, un recién nacido en su desarrollo inicial, sus procesos de regulación metabólico tienen una preponderancia en su estado global, pero se encuentran influenciados, y progresivamente con mayor intensidad, por ciclos de intercambio intersubjetivo con su madre o adulto responsable (diálogo tónico-afectivo Wallon, 1995).

De tal manera, el comportamiento no es determinado (aunque sí condicionado) por la influencia del medio ambiente ni por los procesos fisiológicos que ocurren al interior del individuo, sino por las necesidades y su consecuente comportamiento que se presenta para satisfacer dichas necesidades, como también a partir de la emocionalidad que la persona vive en su particular contexto y situación, en función a las características de comportamiento que se expresan más o menos en forma constante en su proceso histórico de vida. En este sentido, la intencionalidad se estructura hacia la posibilidad y condición de construir e interpretar lo que se es desde la acción. Vale decir, estructurar un camino (comportamiento) que al mismo tiempo va haciendo al caminante. Lo particular es, precisamente, que la dirección o el contenido se generan en el emprendimiento y en sus ajustes ecológicos con el entorno en que se sitúa la acción.

Esto expresa que la acción, como forma concreta del comportamiento, se organiza a partir de procesos intencionados desde la persona en relación con el entorno y medio que habita.

Por consecuencia, en las acciones y el comportamiento, entendido como una continuidad de acciones con un gran propósito general convocador y común, los elementos del medio ambiente adquieren sentido y presencia en la medida que la persona los manipule (en el sentido amplio del termino), y los distinga de acuerdo a algún tipo de sentido o valor de carácter afectivo-simbólico (Damasio, 1999).

De ahí se hace comprensible el hecho que las personas difieran siempre en su comportamiento frente a situaciones iguales, pues se expresa los procesos de evaluación y asignación de valor y sentido a estas situaciones, fuertemente condicionados por el carácter social que ha acontecido en la acción y desde ahí se produce una nueva acción. Estos procesos de evaluación y asignación de sentido a partir de las necesidades, la relacionalidad y la emoción de la persona puede ser más o menos consciente, dependerá de la cultura y las opciones que en ella se enfaticen. Es decir, existe una autonomía relacional, una mutua dependencia entre sujeto y cultura-entorno.

Ahora bien, cómo se puede operacionalizar esta intencionalidad en términos concretos y susceptibles de ser descritos, analizados y comprendidos. En este derroteros se sitúan los niveles de intencionalidad que se describen a continuación. 


\subsection{NIVELES DE LA INTENCIONALIDAD}

De acuerdo a los tipos y niveles de concreción de la acción se puede definir tres momentos de la intencionalidad (Gabler, 1996), a saber:

a. La acción se orienta hacia una meta concreta que provoca un resultado determinado, por ejemplo un jugador de fútbol que patea un balón. En este caso la meta es precisamente patear el balón que tiene como resultado enviar el balón hacia un determinado lugar.

b. Detrás de la meta de la acción existe un objetivo. Siguiendo el ejemplo anterior, éste puede ser convertir un gol, una jugada defensiva o un pase. En este caso el objetivo produce un determinado efecto, que en el caso del jugador o equipo logre ganar.

c. A través de los dos aspectos mencionados anteriormente no se puede determinar el sentido de la acción, aunque se encuentra presente y responde al valor que la acción tiene para la persona que la realiza. En el caso del jugador de fútbol, las acciones de despejar o patear el balón representan, eventualmente, una adecuada labor como futbolista, lo que a su vez implica reconocimiento y valoración de sus pares.

Estos tres niveles de concreción de la acción (Figura 3) responden a tres preguntas básicas:

a.- ¿Hacia cuál resultado concreto se debe conducir el comportamiento, es decir, cuál es la meta a alcanzar?

b.- ¿Cuál es el resultado que se quiere alcanzar, es decir, qué objetivos se persiguen con el resultado?

c.- ¿Qué sentido tiene todo esto en el fondo, por ejemplo, rendir pruebas, participar en competencias, obtener un título profesional? Esta pregunta se relaciona con la escala de valores de cada cual.

Desde de las consideraciones anteriormente descritas, se puede inferir que la pregunta principal sobre el comportamiento o el actuar de las personas no se funda en saber el por qué de ese comportamiento o actuar, sino para qué se comporta o actúa de esa forma.

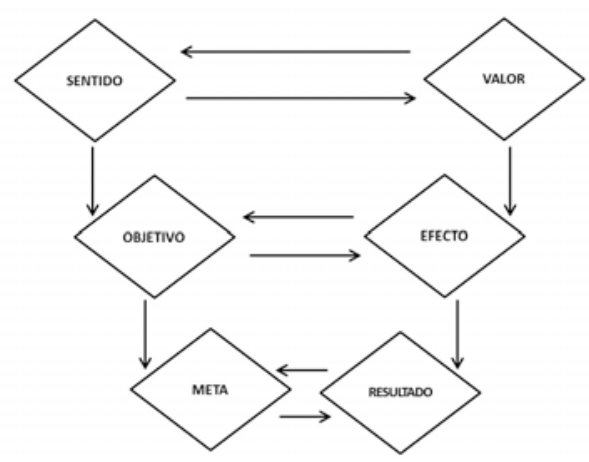

Figura 3. Diagrama de los tres niveles de intencionalidad (Gabler, 1996)

Una vez comprendido el proceso de la acción como proceso intencional, se hace necesario revisar cómo se expresa la construcción de la acción desde su funcionalidades en el comportamiento humano. 


\section{FUNCIONES DE LA ACCIÓN}

El actuar, como proceso intencional permite que se pueda desprender funciones inherentes a dicho proceso que hacen posible lo hasta aquí desarrollado. Dichas funciones van más allá de la realización de una determinada tarea, sea ésta de trabajo, recreativa o de aprendizaje, más bien se orienta hacia una propiedad constitutiva de la misma y que favorece tanto su particularidad como la emergencia de procesos de individuación (Simondon, 2009) y apropiación de la cultura. En dichas funciones se da cuenta de la construcción de lo que hemos llamado como autonomía relacional, pues al mismo momento que tiene lugar el agente autónomo y autopoietico (Maturana y Varela, 1996) tiene lugar la relación. Solo en la convergencia de dos configuraciones es que se genera la identidad y la comunidad. De esta manera, Gabler (1996) propone como mínimo tres funciones fundamentales de la acción, a saber:

6.1. La función exploratoria: En cada acción se explora, se conoce y reconoce la realidad. A través de la información que se recoge en el actuar se hace posible la experiencia, y ésta permanece para la construcción, la evaluación, estabilidad o alteridad de los conceptos, de sí mismo (capacidades, habilidades, aptitudes, representaciones) y sobre su entorno. Este aspecto es especialmente acentuado en juegos, aprendizajes o cualquier acción que se realice por primera vez.

6.2. Función constructiva: En el actuar se crea, construye y modifica la realidad, es decir, a través del actuar son realizadas tareas concretas que implican un impacto directo sobre el entorno lo que implica una modificación del mismo. Al modificarse el medio a través de la acción es posible la creación de nuevas situaciones, objetos, cosas, conceptos y acciones. Este aspecto está enfatizado en las acciones de trabajo, del deporte, el arte, la recreación.

6.3. La función representativa: En el actuar se representa la condición e identidad (personal y social), en ella se expresa cómo es una persona y también cómo ella interpreta su entorno. Al mismo tiempo se presenta, en el actuar, cómo la persona quiere o desea ser vista por los demás (entorno social). En este sentido, el actuar es al mismo tiempo una presentación de sí , como una apropiación y encarnación de las normas, valores y reglas que están presentes en el entorno social. Este aspecto se grafica en las distintas características que se le asignan a los roles. Por ejemplo, ser profesor implica cierta forma de relacionarse, vestirse, hablar, escribir, en fin de comportarse.

A partir de lo señalado, debiéramos entender que las distintas funciones siempre están unidas aunque en determinadas circunstancias y contextos se encuentre una más enfatizada que las demás.

\section{EL ACTUAR COMO COMPORTAMIENTO DE REGULACIÓN COMPLEJO}

Las personas no reaccionan mecánicamente a diferentes estímulos que se le anteponen, sino que frente a objetos que son percibidos e interpretados en forma íntegra a través de procesos complejos al interior de la persona. En consecuencia, la relación 
con el medio ambiente o entorno se traduce en una interacción permanente, no en una influencia directa o determinismo.

Esta relación con el entorno es de carácter complejo, afecta a la persona en sus distintas dimensiones, es decir, lo corporal, concretamente en lo fisiológico, se ve influenciado por el entorno, pero ese efecto no es absoluto, pues operan procesos selectivos de percepción y de interpretación que obedecen a lo señalado el apartado de la intencionalidad de la acción. Pues en definitiva, dependerá fundamentalmente de los propósitos e intenciones, que son formulados desde la dimensión sicológica, qué estímulos y cómo afectan a la persona, condicionados por las dimensiones enfatizadas e involucradas.

Este marco de regulación sicológica dependerá de la historia, del presente y de la realización en sí misma de la acción, es decir a partir de la acción consumada se revisan los procesos sicológicos que hicieron posible la realización de la acción y el grado de efectividad que se logró, de acuerdo a los propósitos e intenciones de la misma . Dichos procesos de regulación sicológica se relacionan con la percepción de la acción en curso, del entorno, la visualización, la ejecución y la evaluación de la misma. Cabe destacar que dichos procesos se realizan de manera no consciente para la persona durante la acción. Este aspecto es sustantivo en el contexto de los actuales conocimientos referidos a lo que se hace, vale decir, la información en si misma se dimensiona como conocimiento en el momento de la experiencia situada directamente con la información referida, de lo contrario se transforma en otra experiencia diferente. Un ejemplo claro es la memorización de una información, normalmente lo que se aprende es a memorizar, y el contenido se va modificando en virtud de las exigencias o de la contingencia. De igual forma ocurre con el aprendizaje de una técnica descontextualizada como un lanzamiento, un determinado golpe o gesto propio de un deporte. En el momento de ocuparlo en un contexto de juego, es altamente probable que no se realice adecuadamente o definitivamente no se produzca.

En este sentido, el mismo proceso de inteligencia se entiende como un producto de la regulación o una constatación de la misma. Pues, al inteligir lo que una cosa realmente es en su situación, diremos que la intelección es verdadera. Verdad sería, pura y simplemente, el momento de la real presencia intelectiva de la cosa en un determinado contexto y a so le llamaríamos realidad (Zubiri, cit. en Dussel, 2007: 111). Dicho proceso ocurre en las dinámicas relaciones que tiene un correlato neurofisiológico y que nos permite la experiencia de lo inteligeible y presente; en tal sentido, los aspectos neurales de la acción también se revelan cono importantes en el momento de la comprensión del proceso psicológico de la acción.

\section{EL ACTUAR COMO FENÓMENO NEURONAL... LAS EMOCIONES Y LOS DEMÁS}

Se ha discutido bastante sobre el aporte de las ultimas décadas que las neurociencias han realizado a la comprensión y explicación de los procesos de actuación y aprendizaje dentro del fenómeno humano (Edelman, 1990, Llinas 2004; Damasio, 1999, 2010; Noë 2010; Rizzolatti, 2006; Fuchs, 2010, por citar algunos). Dichos autores y programas investigativos han abarcado no sólo las descripciones o explicaciones funcionales más tradicionales, sino que se han adentrado hacia los procesos de subjetivación o individuación. Como producto de esta esfuerzo, conceptos como conciencia, subjetividad, meditación, 
espiritualidad no aparecen como campos prohibidos o ajenos al esfuerzo de la ciencia $y$, por consecuencia, dominios que aportan sustantivamente hacia el conocimiento de lo humano y desde sus bases biológicas y sus relaciones con los aspectos culturales.

Precisamente porque el accionar humano se impregna en dichos campos es que se ha pretendido dar cuenta de tales fenómenos sin descuidar, tanto el procedimiento metodológico científico como la experiencia de la acción propiamente tal. Pues el ponerse como una realidad subjetiva, corporal, un self (sí mismo), ante una cosa real, supone un nivel superior de estructura cerebral, "ningún animal puede tener esta autoconciencia lingüística diferenciada entre el "yo bebo" lo ante mi, un vaso de agua real y la conciencia de sí. El mismo hacer-"se" cargo de la realidad presupone dicha autoconciencia" (Dussel, 2007: 113). En este sentido, el comportamiento humano posee ciertas características que le permitiría apropiarse del mundo que genera desde la acción y, simultáneamente, configurarse a sí mismo. Tal condición se traduce en una capacidad cerebral que le permite reconocer y distinguir en una red distribuida de conexiones centrales y periféricas ciclos de regulación metabólica, despliegue senso-motor y reciprocidades intersubjetivas (Varela, 2000; Toro, 2010), coordinadas desde lo neurológico, que permiten la experiencia de lo sensorial y el acto en un mismo momento.

En esta dirección, el aporte de Rizzolatti sobre las "neuronas espejo" o F5, ${ }^{2}$ ha sido sustancial para comprender que los procesos de acción se despliegan en el paralelismo de los canales aferentes y eferentes. En sus palabras:

El hecho de que la información sensorial y la motora sean reducibles a un formato común, codificado por específicos circuitos parietofrontales sugiere que, más allá de la organización de nuestros comportamientos motores, también ciertos procesos generalmente considerados de orden superior y atribuidos a sistemas de tipo cognitivo, como son, por ejemplo, la percepción y el reconocimiento de los actos ajenos, la imitación y las mismas formas de comunicación gestuales o vocales, puedan remitir al sistema motor y encontrar en él su propio sustrato neural primario (2006: 31).

De manera que es desde la acción dentro de un grupo que se va configurando como patrones de comportamiento en una determinada base cultural el sentido y significado de los mismos, una descarga neuronal en dicha zonas referencian no sólo a un gesto o desplazamiento de un objeto en el espacio, sino que se entraman en la generación de sentido. "En otros términos, es como si las neuronas de la Zona F5 y AIP 3 reaccionaran no al simple estímulo como tal, es decir, a su forma o aspecto sensorial, sino también al significado que éste encierra para el sujeto en acción -y reaccionar a un significado equivale a comprender" (Petit, en Rizzolatti, 2006: 57).

Entonces la acción, desde el punto de vista neural, se constituye en la unidad del acto y sentido. Si tuviéramos que hacer el esfuerzo tradicional de centralizar el conocer

2 Las neuronas espejo se denomina a una zona del cerebro ubicada en la corteza frontal agranular que es simbolizada por la letra F y subdividida en de acuerdo numeración arábica de F1 a F7. Dicha nomenclatura, segun Rizzolatti (2006), viene de Von Economo y Koschinas. Tambien es conocida por otras denominaciones. Es interesante mencionar que dicha neuronas según Iacoboni (2008) se caracterizan por permitirnos la posibilidad de leer las acciones y a partir de esos sus estados emocionales, es decir son condición básica de la empatía. Este proceso neuronal consiste básicamente en la activación de zonas senso-motoras equivalentes en un sujeto observador de una acción de un sujeto observado, con mayor intensidad en la medida que se haya experimentado la acción observada.

3 Zona Intraparietal Anterior, de acuerdo a la convención mencionada en el punto 2. 
como una propiedad del cerebro, "la realidad de suyo", de la cosa real no es igual a la existencia intencional de su "re-construcción" en el cerebro. Más bien, es metafórica y emerge desde las propiedades de lo accionado y situación significante.

La realidad, la entidad y fenomenalida expresan, entonces, diversos niveles. La realidad de las cosas en cuanto tal como objetos y la entidad, por otra parte, es de los entes en cuanto se les descubre en relación a su contenido esencial (Dussel, 2007); por último, la fenomenalidad es igualmente de los entes, pero en cuanto se les constituye en su estructura relacional eidética o como sentido mundano.

El cerebro "conoce", en este sentido, al "constituir" el objeto interior de la cosa real exterior. Es una "constitución cognitiva de cosa-objeto que además utiliza la memoria de constituciones pasadas, por lo que Edelman (1990) se refiere a un presente evocado. Conocer, primigeniamente es la presencia intencional o neuronal misma de la cosa real en el cerebro por la acción desplegada en sí misma. El cerebro (por dentro desde un órgano tradicionalmente reconocido como cognoscente), conoce en el proceso de fabricación de lo conociendo(se). Por lo mismo, no es él en sí que conoce, sino todo el sistema humano, y se produce simultáneamente o ipso facto en la acción desplegada.

Por ello, el concepto objetivo como constructo cerebral es ya concepto subjetivo como acto cognitivo: son exactamente lo mismo, aunque con diversas "diferencias" ( el concepto "objetivo" dice referencia a la cosa real, el concepto "subjetivo" al sujeto mismo). Además la categoría de lo "intencional" para los fenomenólogos indica la realidad física "neuronal" (química, orgánica, eléctrica, etc) del ámbito y actividades del cerebro (Dussel, 2007: 118).

Por lo cual, cuando hablamos de aprendizaje verdadero o de criterios de verdad que permiten distinguir y dar cuenta de una situación de verdad, es por la correspondencia de distintos niveles de impresión y despliegue de la acción. Desde lo neuronal hasta lo experiencial propiamente tal, "la verdad es un modo (el modo adecuado) de la captación, actualización, impresión, presencia intencional, neuronal, cerebral, subjetal, relacional de la cosa real" (Ibíd., 118). Se produce por niveles consistentes de coherencia intersubjetiva, que no permite el acceso certero e inefable sino siempre está cargado de apreciación y fuga.

En la realidad de la actualización cerebral de las cosas reales no hay nada "directo" ni "inmediato" sino que todo está directamente mediado por una totalidad mundana (de inmensa complejidad de grupos neuronales en el cerebro), ontológica, biográfica, psicológica, inconsciente, lingüística, cultural, histórica, etc. (Ibíd., 119).

De acuerdo con Edelman (1990), "el sistema afectivo-evaluativo es un momento constitutivo originario del acto mismo de la captación empírica por excelencia del categorizar (...) así como el sistema afectivo-evaluativo constituye un momento del proceso de categorización, de la misma manera la categorización conceptual reorganiza el sistema de valores ordenándolos por su parte, también basándose en el criterio de la 'sobrevivencia', para posteriormente ser subsumido en criterios lingüístico-culturales” (127).

La autoconciencia solo puede darse cuando la corporalidad por las funciones superiores del encéfalo, llamada mente, se comprende y puede nombrarse como mi "yo" (self) o un "nosotros", claramente distinto del "no-yo". Así surge la posibilidad de la reflexión del sujeto sobre sí mismo, tomando, en el mismo proceso, conciencia del estar conociendo como un sujeto cognoscente, y de pertenecer a una comunidad de los hablantes distinta de una mera realidad exterior, desde el horizonte del pasado re-cordado lexicalmente en 
cuanto recurso de una lengua, de una cultura, cuyo horizonte de sentido se vuelca sobre el flujo emocional que en dicha comunidad se estructura coordinadamente.

Emociones como la alegría, tristeza, temor o disgusto corresponden a "estados corporales" que se experimentan y se expresan, en la comportamiento, tanto como el "sentir" felicidad o angustia, que están conectadas a las "señales" o marcadores somáticos (Damasio, 1999, 2010) igual que la euforia, melancolía o pánico. Desde ellos y en la coordinación con los procesos emocionales de los pares y de otras especies se genera la dinámica relacional del lenguaje, tanto formal como informal.

Sin embargo, desde un punto de vista neurológico cerebral, ambos sistemas, es decir, el afectivo y el categorial, tienen la misma necesidad objetiva: responden en primer lugar a las exigencias de la vida (necesidad objetiva primera); en segundo lugar, se articula con el nivel lingüístico-cultural e histórico; y en tercer lugar, como veremos, responden a las exigencias superiores y culturales, universales de una ética crítica (integrada a las funciones del sistema límbico o base del cerebro, junto al lóbulo frontal del córtex).

Este saber que conocemos, profundamente biológico y cultural, conlleva una ética que no podemos soslayar: la aceptación del otro en la convivencia, como el fundamento biológico del fenómeno social, por ende, cualquier cosa que destruya o limite la aceptación del otro (...) desde la competencia hasta la posesión de la verdad, pasando por la certidumbre ideológica, destruye o limita el que se dé el fenómeno social, y por tanto lo humano, porque destruye el proceso biológico que lo genera (Maturana, Cit. en Dussel 2007: 135).

En palabras de Freeman (2007), el conocimiento no es una representación del mundo, es la propia actividad creativa y dinámica, tanto cortical como periférica. Es un proceso de creación continuo y no se puede inyectar, o pasar, sino sólo favorecer. Pero que al mismo tiempo se genera en la relación con los semejantes y diversos, es decir el conocimiento como su proceso de generación o apropiación, a saber es un proceso social, sustentado biológicamente que se actualiza en su propio despliegue. Una forma de entender este proceso es la propuesta que revisaremos a continuación.

\section{LA ENACCIÓN ${ }^{4}$}

Varela $(1996,2000)$ ha propuesto este neologismo para referir que precisamente a partir de los diferentes hallazgos en neurofenomenología ha podido describir que los procesos de cognición y, por ende, de inteligencia se generan en-la-acción, en el hacer. No se refiere al concepto "práctico" muy utilizado dentro de la educación en general y de la educación física en particular. Más bien refiere a que lo que conocemos, se genera en el acoplamiento de nuestro actuar con las contingencias y el entorno en que nos encontramos en cada momento. Así, la acción naturalmente está sometida a ciertos ciclos de operación (Palacios, 2005), a saber: de regulación metabólica, de acoplamiento senso-motor y de regulación intersubjetiva.

4 Es un neologismo propuesto por Varela y que tiene su orienta en el vocablo ingles to enact, referido fundamentalmente al representar un papel o actuar. Se refiere a los procesos de pliegue/despliegue de un actor (u organismo) y su mundo revelado a través de regularidades o patrones que se expresan en diferentes propiedades y situaciones, y que en definitiva nos permiten el conocer. Cf. Varela, 1996. 
El primero de ellos se orienta a que toda acción humana está sustentada en los procesos de homeostasis o dinamoestasis (Varela, 2000), dado que los procesos bioquímicos y físicos, como de regulación y correlación sistémica en el ser humano como organismo, son profundamente dinámicos y sensibles. Los ciclos de regulación de sueño y vigilia, como las relaciones entre procesos de presencia, gasto y suficiencia energética y, de igual forma, los niveles de emoción, condición endocrina e inmunológica, son claros y evidentes (Varela, 2000; Palacios 2005).

En segundo lugar, lo ciclos de acoplamiento senso-motor es lo que históricamente se ha denominado como movimiento, pero que en este trabajo hemos situado como una intencionalidad en curso o en despliegue, pues un organismo se mueve en función de lo que siente y siente en función de como se mueve. Es decir, se encuentra en una tensión interna que le conduce y dirige en una determinada trayectoria. Tal proceso en un entorno siempre dinámico e inestable genera neurodinámicas siempre creativas u originales en alguna medida, aunque nunca absolutamente novedosas. Como ya hemos visto los patrones de acción se estabilizan en la medida de la recurrencia y los contextos de acción de la cultura. En ese per curso se presenta, tanto el sentido de sí mismo, como el sentido de agente o actor (Gallagher, 2010).

El últimio ciclo refiere a que los procesos de conocimiento se generan, como ya se ha mencionado, desde la capacidad de relación y lectura del estado emocional-psicológico de los demás, fundamentalmente de la propia especie, pero en el caso de los mamíferos superiores (con un énfasis en el caso humano), también de otras. Dicha condición se ha denominado como empatía. ${ }^{5}$ Como nos dice Noë, "podemos rechazar la idea de que somos islas autónomas que tomamos decisiones en base a un escaneo escrupuloso y a un juicio sano. Nuestra naturaleza está mucho más íntimamente enredada con el entorno" (2011: 129). De manera que la acción es siempre vinculada y vinculante tanto a la historia cultural como a la situación y contingencia relacional.

\section{CONCLUSIÓN}

Desde los diferentes argumentos presentados podemos establecer qué acción es la unidad del comportamiento humano, que puede ser definida como intencionalidad en despliegue. Es decir, ocurre a partir de un determinado sentido a expresar y/o conseguir. Tal situación tiene enormes repercusiones en todas la áreas de desarrollo y estudio de lo humano, toda vez que le otorga a lo humano la potencialidad de asumir o tomar para sí su propia confluencia en el entorno y en sí mismo. Recae, por lo tanto, en él/ella la

\footnotetext{
Se comprende la empatía en el sentido biológico-etológico del primatólogo Franz de Waal (2011), quien sostiene que la capacidad de co-sentir está muy presente en los animales en general, pero que en el caso de los mamíferos, aún mas en los primates y con gran distinción en los humanos. En este sentido, la posibilidad de la captación del estado subjetivo de un semejante se constituye en una capacidad estructural de la vida animal en general y especialmente de la humana. Esta última con componentes lingüísticos de alta complejidad y especificación que permite recobrar o a lo menos caracterizar la condición presente desde los vivido, proyectar consecuencias e intencionar deliberadamente comportamientos propios y ajenos. Del mismo modo, en el caso humano, permite complejas compresiones de la experiencia de los semejantes o seres de otras especies. Por otra parte, desde el punto de vista filosófico, Evan Thompson ha caracterizado la empatía en tres niveles que incluyen los aspectos corporales, psicológicos y culturales (ver Toro, 2005).
} 
factibilidad de decidir de acuerdo a su contexto, en el amplio sentido de la palabra, lo que le permite dar con la realización de su propósito, pero al mismo tiempo, tal situación se genera en las condiciones de acción y de relacionalidad de la misma. A razón de ello, lo multidimensional, la complejidad y la referencia intermodal e intersubjetiva del actuar, constituyen un fenómeno que implica o demanda una permanente y multivariada atención, si se pretende desarrollar a través de procesos o ambientes intencionados de aprendizaje del comportamiento.

Llevado al plano de la educación, adquiere una vital importancia adentrarse en las intenciones y sentidos del/a estudiante como en la transparencia de los propósitos del educador, a fin de que el primero conozca y acuerde con el segundo, intenciones que surjan de ambos actores del proceso educativo. De la misma forma, en cualquier tipo de relación se sostiene en la base de compartir determinadas intenciones y propósitos para que se pueda actuar en conjunto frente a determinados desafíos que la misma relación demanda.

Mirado desde el paradigma de la Motricidad Humana (Sergio, 1999, 2008), cabe señalar que la acción es la unidad de análisis que permite establecer lo que un sujeto/a operativiza, de acuerdo a una intención en su vivencia cotidiana o especial (lúdica, ergonómica o mixta) lo que implica el desencadenamiento de una red de identidades (musculares, fisiológicas, psicológicas, sociales, entre otras) que constituyen una dimensión de la acción, pero que no tienen sentido aisladamente sino dentro de la configuración y unidad de la misma, en el momento de ser estudiadas y comprendidas. En tal sentido, el movimiento no es una característica humana sino de los objetos y cuerpos desprovistos de intencionalidad. Lo mismo que expresarse en términos de un componente de la acción (física, espiritual, mental o como se quiera diferenciar). Por el contrario, una definición o denominación dependerá del sentido o propósito de la misma acción. Por ejemplo, una actividad o ejercicio puede ser saludable, deportiva, recreativa, laboral, lúdica, dancística, entre muchas más.

Esto parece ser una obviedad, pero mas allá de lo nominal, y sin renunciar a ello, lo que se busca es la pertinencia y el reconocimiento de que una acción está lejos de ser un desplazamiento morfo-funcional o la adaptación a un estímulo, sino más bien a una relación sustentada en factores de eco-auto-organización, basadas en la experiencia directa de un sí mismo, que es agente y en constante referencia cruzada con otros de su misma especie, o de otras, en una determinada situación témporo-espacial. Al comprender la acción desde una perspectiva ecológica, se le otorga a los objetos una cualidad de actuantes (no actores), es decir, afectan sobre el ser humano desprovistos de toda intencionalidad, no obstante, tienen un impacto en la percepción y actuación del ser humano. Es importante destacar que esto no debe confundirse con un determinismo conductista de acuerdo al medio en el cual se vive, sino que se relaciona con el efecto que tienen los objetos, cosas o seres vivos y ambientes sociales o naturales dentro de la realización y desarrollo de una acción, en consecuencia, dentro del comportamiento del ser humano.

En el sentido de la proyección del estudio sobre la acción, quisiéramos señalar el interesante aporte que plantea Francisco Varela a través del concepto de enacción, que sugiere, en términos generales, la unidad de la consciencia y la acción durante todo el proceso de realización de la misma y no sólo en la etapa de anticipación y evaluación. Varela (2000) realiza una interesante y concluyente fusión de la acción, desde la fenomenología con la tradición vivencial del budismo, lo que a simple vista parece una 
excentricidad, resulta un importante aporte a la discusión sobre todo desde la praxis de un neurobiólogo. El horizonte final de dicha vinculación es asumir el desafío de la experiencia como un procesos de conciencia presente y plena, aprovechando, desde el budismo, ciertas herramientas y principios que pueden favorecer el logro o arribo al mencionado horizonte.

Por último, no está demás recordar que la tradición occidental ha marcado profundamente la racionalididad de los sujetos de esta sociedad que se plasma en un exacerbado privilegio de la razón por sobre la corporeidad y la emocionalidad, al considerarlos como remanentes de una animalidad negativa. En este punto, Varela plantea una alternativa que supera largamente el trasnochado enfoque dualista, pero al mismo tiempo entrega importantes luces que nos permiten acercarnos a una conciencia de lo humano más integral. No sólo desde el punto de vista de su unidad en cuanto organismo, sino también como una unidad en su actuar y en su comportamiento con la condición de ser naturalcultural. De la misma forma, desarrolla un enfoque donde se considera y se establece la fundamental relación del ser humano y su sociedad con el entorno natural donde se desarrollan, constituyéndose en una triada acoplada estructuralmente, cuya división es sólo analítica, pues en la enacción, donde emergen los significados y los sentidos, no es posible separarlos ni obviar alguno de estos elementos.

En este contexto, una educación física a la altura de esta consideración ya no sería sólo física, no puramente en términos lingüísticos, sino de propuesta disciplinar y epistémica diferente, basada en el desarrollo situado y político de los actores involucrados, como de su entorno. Enfatizando procesos complejos e integradores, reconociendo la cultura y la capacidad transformativa de la acción. El eje central se desarrolla en el fomento del "gobierno" de sí, de la capacidad de guiarse y desde las propias intencionalidades hacia el encuentro y co-construcción con el entorno-mundo. Tal condición pone en el lugar protagónico la vivencia y el sentido de la misma, la autonomía y la relación y nos exige a lo menos una reformulación conceptual, programática y epistémica, proceso, por cierto, que requiere de mucho rigor y generosidad.

\section{REFERENCIAS BIBLIOGRÁFICAS}

Arendt, H. (1993). La condición humana. Barcelona: Paidos.

Batalla, A. (2005). Retroalimentación y aprendizaje motor: influencia de las acciones realizadas de forma previa a la recepción del conocimiento de los resultados en el aprendizaje retención de habilidades motrices. Tesis de Doctorado presentada en La Facultad de Pedagogía de la Universidad de Barcelona. Barcelona.

Benedetti, M. (2009). Inventario cuatro. Montevideo: Visor Libros.

Changeux, J. (2003). A verdade e o cérebro. O homen de verdade. Lisboa: Instituto Piaget.

Damasio, A. (1999). Sentir lo que sucede. Santiago de Chile: Andrés Bello.

Damasio, A. (2010). Y el cerebro creo al hombre. Barcelona: Ediciones Destino.

Dantas, P. (2003). A intencionalidade do corpo propio. Lisboa: Instituto Piaget.

De Waal, F. (2011). La edad de la empatía. Barcelona: Metatemas.

Dussel, E. (2007). Materiales para una política de la liberación. Ciudad de Mexico: Plaza y Valdés.

Gabler, H. (1996): Einführung in die Sportpsychologie. Schorndorf: Hoffman.

Gallagher, S. y Schmiking, D. (2010) (Edit). Handbook of phenomenology and cognitive sciences. Massachusetts: Springer. 
Edelman, G. (1990). The remebered present: a biological therory of consciusness. New York: Basic Books.

Freeman, W. (2007). Dinámicas no lineales e intencionalidad: El rol de las teorías cerebrales en las ciencias de la mente. En Ibañez, A. y Cosmelli, D., Nuevos enfoques de la cognición -redescubriendo la dinámica de la acción, la intención y la intersubjetividad. Santiago de Chile: Universidad Diego Portales.

Fuchs, T. (2010). Das Gehirn -ein Bezihungsorgan. Eine phänomenologisch-okölogische Konzeption. Sttutgart: Kolhhammer.

Iacoboni, M. (2008). Las neuronas espejo. Empatía, neuropolítica, autismo, imitación,o de cómo entendemos a los otros. Madrid: Katz Conocimiento.

James, W. (2009). Un universo pluralista. Filosofía de la experiencia. Buenos Aires: Cactus.

Kandel, E.; Jessel, T. y Schwartz, J. (2003). Neurociencia y conducta. Madrid: Prentice Hall.

Luckmann, T. (1997). La acción social. Barcelona: Paidos.

Llinas, R. (2004). El cerebro y el mito del yo. Barcelona: Norma.

Maturana, H. (1999). Transformación en la convivencia. Santiago de Chile: Dolmen.

Maturana, H. y Varela, F. (1996). De máquinas a seres vivos. Santiago de Chile: Editorial Universitaria.

Merleau-Ponty, M. (2004). O Olho e o espírito. Sao Paulo: Cosac \& Naify.

Morin, E. (1992). El paradigma perdido. Barcelona: Seix Barral.

Noë, A. (2010). Fuera de la cabeza. Por qué no somos el cerebro y otras lecciones de la biología de la consciencia. Barcelona: Kairos.

Palacios, A. (2005). Complejidad biológica, artificial y enacción. Conferencia Instituto de Sistemas Complejos de Valparaíso. Centro de Neurociencias de Valparaíso.

Pfeifer, R. y Bongard, J. (2007). How the body shapes the way we think. A new view of intelligence. Massachusetts: MIT Press.

Rizzolatti, G. y Sinigalia, C. (2006). Las neuronas espejo. Los mecanismos de la empatía emocional. Barcelona: Paidos Transiciones.

Sergio, M. (1999). Motricidade Humana, um corte epistemológico. Lisboa: Instituto Piaget.

Sergio, M. (2008). Textos insólitos. Lisboa: Instituto Piaget.

Sergio, M. y Toro, S. (2004). Hacia un paradigma de la motricidad humana. Trigo, E.; Jaramillo, G. y Deibar, R. (Org.), Consentido. Popayán: Universidad del Cauca.

Simondon, G. (2009). La individuación. Buenos Aires: Editorial Cactus y la Cebra Ediciones.

Schütz, A. (1995). La construcción significativa del mundo social. Introducción a la sociología comprensiva. Barcelona: Paidos.

Toro, S. (2010). Neurociencias y aprendizaje, un texto en construcción. Estudios Pedagógicos Vol. $36, \mathrm{~N}^{\mathrm{o}} 2,323-341$.

Toro, S. (2009). Memoria y evolución. Pilares de una educación consiente desde la motricidad humana. Profissao Docente, Vol.7

Varela, F. (1996). Conocer. Las ciencias cognitivas: tendencias y perspectivas. Cartografía de las ideas actuales. Barcelona: Gedisa.

Varela, F. (2000). El fenómeno de la vida. Santiago de Chile: Dolmen.

Varela, F.; Thompson, E. y Rosch, E. (2005). De cuerpo presente. Las ciencias cognitivas y la experiencia humana. Barcelona: Gedisa Editorial.

Vayer, P. y Roncin, Ch. (2000). Psicologia das actividades corporais. Lisboa: Instituto Paiget.

Wallon, H. (1995). A evoluçao psicológica da criança. Lisboa: Ediciones 70.

Zîzêk, S. (2011). En defensa de las causas perdidas. Barcelona: Akal.

Zubiri, X. (1986). Sobre el hombre. Madrid: Alianza/ Fundación Xavier Zubiri. 